Теорія Ймовір. та Матем. Статист. Вип. 80, 2009
Theor. Probability and Math. Statist. No. 80,2010 , Pages 71-84 S 0094-9000(2010)00795-2

Article electronically published on August 19, 2010

\title{
THE HEAT EQUATION WITH RANDOM INITIAL CONDITIONS FROM ORLICZ SPACES
}

UDC 519.21

\author{
YU. V. KOZACHENKO AND K. I. VERESH
}

\begin{abstract}
Conditions for justification of the Fourier method for parabolic equations with random initial conditions from Orlicz spaces of random variables are obtained. Bounds for the distribution of the supremum of solutions of such equations are found.
\end{abstract}

We study conditions justifying the application of the Fourier method for parabolic equations with random initial conditions and obtain bounds for the distribution of the supremum of solutions of these equations. Similar problems for hyperbolic equations are considered in [1, 2]. A survey of the corresponding results can be found in [3, 4]. In what follows we consider random initial conditions from the Orlicz spaces of random variables.

The paper is organized as follows. Section 1 contains necessary definitions and results of the theory of the Orlicz space. The setting of the problem as well as statements of the main results of the paper is given in Section 2. Conditions for the convergence of stochastic processes in $C(T)$ and bounds for the distribution of the supremum of solutions of the corresponding equations are presented in Section 3. The proofs of the main results are placed in Section 4

\section{Stochastic Processes Belonging to an Orlicz SPACE}

Definition 1.1 (3). An even, continuous, convex function $U(x)$ such that $U(x)>0$ for $x \neq 0$ is called a $C$-function.

Let $\{\Omega, \Im, P\}$ be a standard probability space.

Definition $1.2(4)$. The space $L_{U}(\Omega)$ of random variables $\xi(\omega)=\xi, \omega \in \Omega$, is called the Orlicz space generated by a $C$-function $U(x)$ if, for any $\xi \in L_{U}(\Omega)$, there exists a constant $r_{\xi}$ such that $\mathrm{E} U\left(\xi / r_{\xi}\right)<\infty$.

The Orlicz space $L_{U}(\Omega)$ is a Banach space with respect to the norm

$$
\|\xi\|_{L_{U}}=\inf \left\{r>0: \mathrm{E} U\left(\frac{\xi}{r}\right) \leq 1\right\} .
$$

Definition 1.3. Let $X(t)=\{X(t), t \in T\}$ be a stochastic process. We say that $X$ belongs to the Orlicz space $L_{U}(\Omega)$ if, for all $t \in T$, the random variable $X(t)$ belongs to the space $L_{U}(\Omega)$.

Lemma $1.1(3)$. Let $\xi \in L_{U}(\Omega)$ and let $\mathrm{E} U(\xi / r) \leq a$ for some $r>0$ and $a>0$. Then $\|\xi\|_{L_{U}} \leq r \max \{0 ; a\}$.

2000 Mathematics Subject Classification. Primary 60G60, 60G17.

Key words and phrases. Parabolic equations, Orlicz spaces. 
Lemma $1.2([3])$. If $\xi \in L_{U}(\Omega)$, then, for all $x>0$,

$$
\mathrm{P}\{|\xi|>x\} \leq \frac{1}{U\left(\frac{x}{\|\xi\|_{L_{U}}}\right)} .
$$

Definition 1.4. We say that a $C$-function $U$ is subordinate to a $C$-function $\mathcal{V}$ and denote $U \prec \mathcal{V}$ if there exist two numbers $x_{0} \geq 0$ and $C>0$ such that $U(x) \leq \mathcal{V}(C x)$ for all $x$ such that $|x|>x_{0}$. We say that two $C$-functions $U(x)$ and $\mathcal{V}(x)$ are equivalent if $U(x) \prec \mathcal{V}(x)$ and $\mathcal{V}(x) \prec U(x)$.

Theorem $1.1([3])$. Let a $C$-function $U$ be subordinate to a $C$-function $\mathcal{V}$. Then

$$
L_{\mathcal{V}}(\Omega) \subset L_{U}(\Omega)
$$

and there exists a constant $K$ such that

$$
\|\xi\|_{L_{U}} \leq K\|\xi\|_{L_{\mathcal{V}}}
$$

for all $\xi \in L_{\mathcal{V}}(\Omega)$. If two $C$-functions $U(x)$ and $\mathcal{V}(x)$ are equivalent, then $L_{U}(\Omega)=L_{\mathcal{V}}(\Omega)$ and the norms $\|\cdot\|_{L_{U}}$ and $\|\cdot\|_{L_{\mathcal{V}}}$ are equivalent, too.

Definition $1.5([5])$. Let $U(x)$ be a $C$-function such that $\mathcal{V}(x)=x^{2}$ is subordinate to the function $U(x)$. A family $\Delta$ of centered random variables $(\mathrm{E} \xi=0, \xi \in \Delta)$ from the Orlicz space $L_{U}(\Omega)$ is called a strictly Orlicz family if there exists a constant $C_{\Delta}$ such that

$$
\left\|\sum_{i \in I} \lambda_{i} \xi_{i}\right\|_{L_{U}} \leq C_{\Delta}\left(\mathrm{E}\left(\sum_{i \in I} \lambda_{i} \xi_{i}\right)^{2}\right)^{1 / 2}
$$

for all finite collections of random variables $\xi_{i} \in \Delta, i \in I$, and for all $\lambda_{i} \in \mathbb{R}^{1}, i \in I$.

Definition 1.6. A stochastic process $x=\{x(t), t \in T\}, x \in L_{U}(\Omega)$, is called a strictly Orlicz process if the collection of the random variables $x=\{x(t), t \in T\}$ is a strictly Orlicz family. Two stochastic processes $x=\{x(t), t \in T\}$ and $y=\{y(t), t \in T\}$ are called jointly strictly Orlicz processes if the collection of the random variables $\{x(t), y(t), t \in T\}$ is a strictly Orlicz family.

Definition 1.7 ([3]). We say that the $g$-condition holds for a $C$-function $U$ if there are some constants $z_{0} \geq 0, k>0$, and $A>0$ such that

$$
U(x) U(y) \leq A U(k x y)
$$

for all $x \geq z_{0}$ and $y>z_{0}$.

\section{Setting of the Problem And main Results}

Consider a boundary value problem for a parabolic equation with two independent variables $0 \leq x \leq \pi$ and $t \geq 0$, namely

$$
\begin{gathered}
\frac{\partial}{\partial x}\left(p \frac{\partial V}{\partial x}\right)-q V-\rho \frac{\partial V}{\partial t}=0, \\
V(t, 0)=0, \quad V(t, \pi)=0, \\
\left.V(x, t)\right|_{t=0}=\xi(x),
\end{gathered}
$$

where $\xi(x)$ is a continuous with probability one stochastic process belonging to the Orlicz space $L_{U}(\Omega)$.

The functions $p=(p(x), x \in[0, \pi]), q=(q(x), x \in[0, \pi])$, and $\rho=(\rho(x), x \in[0, \pi])$ in equation (2) are such that

1) $p(x)>0, q(x) \geq 0$, and $\rho(x)>0$ for all $x \in[0, \pi]$

2) $\rho(x)$ and $p(x)$ are twice continuously differentiable functions on $[0, \pi]$; 
3) $q(x)$ is a continuously differentiable function on $[0, \pi]$.

We now state the two main results of the paper.

Theorem 2.1. Let the initial condition $\xi=\{\xi(x), x \in[0, \pi]\}$ on the right hand side of (41) be a strictly Orlicz stochastic process belonging to the Orlicz space $L_{U}(\Omega)$ of random variables, where $U(x)$ is a C-function such that the function $V(x)=x^{2}$ is subordinate to $U(x)$ and condition $g$ holds for $U(x)$. Assume that the stochastic process $\xi$ is separable and mean square continuous, $\mathrm{E} \xi(x)=0$, and $\mathrm{E} \xi(x) \xi(y)=B(x, y)$. Let

$$
V(t, x)=\sum_{k=1}^{\infty} \xi_{k} e^{-\lambda_{k} t} X_{k}(x)
$$

where $X_{k}(x)$ are eigenfunctions and $\lambda_{k}$ are eigenvalues of the Sturm-Liouville problem

$$
\begin{gathered}
L(v)=\frac{d}{d x}\left(p \frac{d v}{d x}\right)-q v+\lambda \rho v=0, \\
v(0)=0, \quad v(\pi)=0, \\
\xi_{k}=\int_{0}^{\pi} X_{k}(x) \xi(x) \rho(x) d x .
\end{gathered}
$$

Let there exist a continuous increasing function $\varphi=\{\varphi(\lambda), \lambda>0\}$ such that $\varphi(\lambda)>0$ for $\lambda>0$ and $\Psi(\lambda)=\lambda / \varphi(\lambda), \lambda>0$, increases for $\lambda \geq v_{0}$, where $v_{0}$ is a certain constant. We also assume that

$$
\sup _{|x-y| \leq h}\left(\mathrm{E}(\xi(x)-\xi(y))^{2}\right)^{1 / 2} \leq C\left(\varphi\left(\frac{1}{h}+v_{0}\right)\right)^{-1} .
$$

Moreover, let

$$
\int_{0}^{\varepsilon} U^{(-1)}\left(\frac{\pi}{2}\left(\varphi^{(-1)}\left(\frac{c}{v}\right)-v_{0}\right)+1\right) d v<\infty
$$

for all $\varepsilon>0$ and $c>0$.

If the series

$$
\sum_{k=1}^{\infty} \sum_{l=1}^{\infty}\left|\mathrm{E} \xi_{k} \xi_{l}\right| \varphi\left(\lambda_{k}+v_{0}\right) \varphi\left(\lambda_{l}+v_{0}\right)
$$

converges, then, for all $\sigma>0$, the series

$$
\sum_{k=1}^{\infty} \xi_{k} e^{-\lambda_{k} t} \lambda_{k}^{m} X_{k}^{(s)}(x)=S_{m s}(t, x)
$$

converges with probability one and uniformly in $0 \leq x \leq \pi$ and $t \geq \sigma$, where $X_{k}^{(s)}(x)$ denotes the derivative of order $s$ in $x$. (The following combinations of the parameters $s$ and $m$ are possible: $s \in\{0,1,2\}$ if $m=0$ or $s=0$ if $m=1$; the corresponding series are the derivatives in $t$ and $x$ of orders $m$ and $s$, respectively, of the random function $V(t, x)$ defined by the right hand side of (55).)

Assume further that the function $V(t, s)$ satisfies with probability one equation (2) in the domain $0 \leq x \leq \pi, t \geq \sigma$ and that condition 2) holds. Moreover let

$$
V(t, x) \rightarrow \xi(x) \quad \text { as } t \rightarrow 0
$$

in probability and uniformly with respect to $x \in[0, \pi]$.

If the function $\varphi$ is such that

$$
\int_{0}^{\varepsilon} U^{(-1)}\left(\left(\frac{\pi}{2}\left(\varphi^{(-1)}\left(\frac{c}{v}\right)-v_{0}\right)+1\right)^{2}\right) d v<\infty
$$


for all $\varepsilon>0$ and $c>0$ and moreover

$$
\sum_{k=1}^{\infty} \sum_{l=1}^{\infty}\left|\mathrm{E} \xi_{k} \xi_{l}\right| \varphi\left(\lambda_{k}+v_{0}\right) \varphi\left(\lambda_{l}+v_{0}\right)<\infty
$$

then $V(t, x) \rightarrow \xi(x)$ with probability one as $t \rightarrow 0$ and uniformly with respect to $x \in[0, \pi]$.

Theorem 2.2. Let the assumptions of Theorem 2.1 hold. Moreover let conditions (11) and (12) be satisfied for the function $\varphi$. Denote $V_{T}=\{0 \leq x \leq \pi, 0 \leq t \leq T\}$. Then

$$
\mathrm{P}\left\{\sup _{t, x \in V_{T}}|V(t, x)|>\varepsilon\right\} \leq\left(U\left(\frac{\varepsilon}{\hat{B}(\theta)}\right)\right)^{-1}
$$

where

$$
\hat{B}(\theta)=\frac{1}{\theta(1-\theta)} \int_{0}^{\omega_{0} \theta} \chi_{U}\left(\left(\frac{\pi}{2}\left[\varphi^{-1}\left(\frac{R}{v}\right)-v_{0}\right]+1\right)\left(\frac{T}{2}\left[\varphi^{-1}\left(\frac{R}{v}\right)-v_{0}\right]+1\right)\right) d v .
$$

Here $\chi_{U}$ is defined by relation (15) below, $R=C_{\Delta} \cdot \sqrt{W} \sqrt{2 \max \left(2 C_{x}, L\right)}$, the meaning of the constant $L$ is explained in Lemma 4.5,

$$
W=\sum_{k=1}^{\infty} \sum_{l=1}^{\infty}\left|\mathrm{E} \xi_{k} \xi_{l}\right| \varphi\left(\lambda_{k}+v_{0}\right) \varphi\left(\lambda_{l}+v_{0}\right)
$$

$C_{\Delta}$ is a constant involved in Definition 1.5, and $C_{X}$ is a constant such that $\left|X_{k}(x)\right|<C_{X}$.

3. Conditions for the CONVERGEnCE in PRobability of STOCHASTIC PRocesses IN $C(T)$ AND BOUNDS FOR THE DISTRIBUTION OF THE SUPREMUM

Theorem 3.1 (Theorem 3.2 in [6]). Let $(T, d)$ be a compact metric space and let

$$
X_{n}=\left\{X_{n}(t), t \in T\right\}
$$

be a sequence of stochastic processes in $L_{U}(\Omega)$. Assume that the $g$-condition is satisfied for the function $U$ and that all the processes $X_{n}(t)$ are separable in $(T, d)$ and that $\rho_{n}(t, s)=\left\|X_{n}(t)-X_{n}(s)\right\|_{L_{u}}$. Let

1) $\rho(t, s) \leq z(d(t, s))$, where $\rho(t, s)=\sup _{n>1} \rho_{n}(t, s)$ and where $z=\{z(x), x>0\}$ is a function such that $z(x) \rightarrow 0$ as $x \rightarrow 0$;

2) $N_{\rho}(u)$ denotes the metric capacity of the space $(T, \rho)$;

3) $\int_{0}^{\varepsilon} U^{(-1)}\left(N_{\rho}(u)\right) d u<\infty$ for all $\varepsilon>0$.

Then, for all $\sigma>0$,

$$
\lim _{\varepsilon \rightarrow 0} \sup _{n \geq 1} P\left\{\sup _{\substack{t, s \in T \\ d(t, s)<\varepsilon}}\left|X_{n}(t)-X_{n}(s)\right|>\sigma\right\}=0 .
$$

Theorem $3.2([3)$. Let $(T, d)$ be a compact metric space and let $C(T)$ be the Banach space of continuous functions equipped with the uniform norm. Let $X_{n}=\left\{X_{n}(t), t \in T\right\}$, $n \geq 1$, be a sequence of separable stochastic processes. If

$1)$ the sequence $\left(X_{n}(t), n \geq 1\right)$ converges in probability for all $t \in T_{S}$, where $T_{S}$ is an arbitrary set that is dense in $T$;

2) for all $\sigma>0$,

$$
\lim _{\varepsilon \rightarrow 0} \sup _{n \geq 1} \mathrm{P}\left\{\sup _{\substack{t, s \in T \\ d(t, s)<\varepsilon}}\left|X_{n}(t)-X_{n}(s)\right|>\sigma\right\}=0,
$$


then all processes $X_{n}(t)$ are continuous with probability one and the sequence $X_{n}(t)$ converges in probability in $C(T)$ to $X(t) \in C(T)$.

Theorem 3.3. Let $\mathbb{R}^{k}$ be the $k$-dimensional Euclidean space,

$$
d(t, s)=\max _{1 \leq i \leq k}\left|t_{i}-s_{i}\right|
$$

$T=\left\{\left(t_{1}, \ldots, t_{k}\right): 0 \leq t_{i} \leq T_{i}, i=1, \ldots, k\right\}$, and let $X_{n}=\left\{X_{n}(t), t \in T\right\}, n=1,2, \ldots$, be a sequence of separable stochastic processes belonging to the Orlicz space $L_{U}(\Omega)$, where the function $U$ satisfies the $g$-condition. Assume that

1) for all $t \in T$,

$$
X_{n}(t) \rightarrow X(t) \quad \text { as } n \rightarrow \infty
$$

2)

in probability;

$$
\sup _{d(t, s) \leq h} \sup _{n \geq 1}\left\|X_{n}(t)-X_{n}(s)\right\| \leq \sigma(h),
$$

where $\sigma=\{\sigma(h), h>0\}$ is a continuous increasing function such that $\sigma(h) \rightarrow 0$ as $h \rightarrow 0$;

3) for some $\varepsilon>0$,

$$
\int_{0}^{\varepsilon} U^{(-1)}\left(\prod_{i=1}^{k}\left(\frac{T_{i}}{2 \sigma^{(-1)}(u)}+1\right)\right) d u<\infty,
$$

where $\sigma^{(-1)}(u)$ is the inverse function to $\sigma(u)$.

Then the processes $X_{n}(t)$ are continuous with probability one and converge in probability in the space $C(T)$.

Proof. The proof of this theorem follows from Theorems 3.1 and 3.2 , since

$$
N_{\rho}(u) \leq \prod_{i=1}^{k}\left(\frac{T_{i}}{2 \sigma^{(-1)}(u)}+1\right) .
$$

Theorem 3.4. Let $(T, \rho)$ be a compact metric space and let $N(u)$ denote the metric capacity of the space $(T, \rho)$, that is, $N(u)$ denotes the minimum number of closed balls of radius $u$ that cover $(T, \rho)$. Assume that $X=\{X(t), t \in T\}$ is a separable stochastic process belonging to the space $L_{U}(\Omega)$, where the function $U$ is such that the $g$-condition holds. Let there exist an increasing continuous function

$$
\sigma=\sigma(h), \quad 0 \leq h \leq \sup _{t, s \in T} \rho(t, s)
$$

such that

$$
\sup _{\rho(t, s) \leq h}\|X(t)-X(s)\|_{U} \leq \sigma(h) .
$$

Assume further that

$$
\int_{0}^{\varepsilon} \chi_{U}\left(N\left(\sigma^{(-1)}(u)\right)\right) d u<\infty
$$

for some $\varepsilon$, where

$$
\chi_{U}(n)= \begin{cases}n, & n<U\left(z_{0}\right), \\ C_{U} U^{(-1)}(n), & n \geq U\left(z_{0}\right)\end{cases}
$$


and where $C_{U}=K\left(1+U\left(z_{0}\right)\right) \max (1, A), z_{0}, k$, and $A$ are the constants involved in Definition 1.7, and $\sigma^{(-1)}(h)$ is the inverse function to $\sigma(h)$. Then the random variable $\sup _{t \in T}|X(t)|$ belongs to the space $L_{U}(\Omega)$ with probability one and

$$
\left\|\sup _{t \in T}|X(t)|\right\|_{U} \leq\left\|X\left(t_{0}\right)\right\|_{U}+\frac{1}{\theta(1-\theta)} \int_{0}^{w_{0} \theta} \chi_{U}\left(N\left(\sigma^{(-1)}(u)\right)\right) d u=B\left(t_{0}, \theta\right),
$$

where $t_{0}$ is an arbitrary point of the set $T$ and $w_{0}=\sigma\left(\sup _{t \in T} \rho\left(t_{0}, t\right)\right), 0<\theta<1$. Moreover,

$$
\mathrm{P}\left\{\sup _{t \in T}|X(t)|>\varepsilon\right\} \leq\left(U\left(\frac{\varepsilon}{B\left(t_{0}, \theta\right)}\right)\right)^{-1}
$$

for all $\varepsilon>0$.

Proof. This theorem is a particular case of Theorem 2.2 and Lemma 2.3 of 7.

Remark 3.1 (7]). Theorem 3.4 remains valid if the number $\omega_{0}$ on the right hand side of (16) is replaced by $2 \sup _{t \in T}\|X(t)\|_{U}$.

Corollary 3.1. Let

$$
T=\{(x, t): 0 \leq x \leq b, c \leq t \leq d\}
$$

and $\rho\left((x, t),\left(x_{1}, t_{1}\right)\right)=\max \left(\left|x-x_{1}\right|,\left|t-t_{1}\right|\right)$ in Theorem 3.4, Further let

$$
\int_{0}^{\varepsilon} U^{(-1)}\left(\left(\frac{b}{2 \sigma^{(-1)}(u)+1}+1\right)\left(\frac{d-c}{2 \sigma^{(-1)}(u)}+1\right)\right) d u<\infty
$$

for some $\varepsilon>0$ and

$$
\begin{aligned}
& B\left(\hat{t}_{0}, \theta\right) \leq \tilde{B}\left(\hat{t}_{0}, \theta\right) \\
& =\left\|X\left(\hat{t}_{0}\right)\right\|_{U}+\frac{1}{\theta(1-\theta)} \int_{0}^{w_{0} \theta} \chi_{U}\left(\left(\frac{b}{2 \sigma^{(-1)}(u)}+1\right)\left(\frac{d-c}{2 \sigma^{(-1)}(u)}+1\right)\right) d u,
\end{aligned}
$$

where $\hat{t}_{0}=\left(x_{0}, t_{0}\right)$. Then condition (14) holds. Moreover

$$
\mathrm{P}\left\{\sup _{t \in T}|X(t)|>\varepsilon\right\} \leq\left(U\left(\frac{\varepsilon}{\tilde{B}\left(t_{0}, \theta\right)}\right)\right)^{-1}
$$

for all $\varepsilon>0$.

Proof. The corollary follows from Theorem 3.4, since

$$
N(u) \leq\left(\frac{b}{2 u}+1\right)\left(\frac{d-c}{2 u}+1\right) .
$$

Remark 3.2. Corollary 3.1 remains valid if the number $\omega_{0}$ on the right hand side of (19) is replaced by $2 \sup _{t \in T}\|X(t)\|_{U}$.

\section{Auxiliary Results and proofs of the main theorems}

Consider the series

$$
\sum_{k=1}^{\infty} \xi_{k} e^{-\lambda_{k} t} \lambda_{k}^{m} X_{k}^{(s)}=S_{m s}(t, x)
$$

for two sets of parameters $s$ and $m$ : either for $s \in\{0,1,2\}$ and $m=0$ or for $s=0$ and $m=1$.

Let $\xi(x), 0 \leq x \leq \pi$, be a strictly Orlicz process such that the series $S_{m s}(t, x)$ converge in the mean square sense for all $0 \leq x \leq \pi$ and $t \geq 0$. Then it follows from [5] that all 
$S_{m s}(t, x)$ are strictly Orlicz stochastic processes in the domain $0 \leq x \leq \pi, 0 \leq t \leq T$, where $T>0$ is an arbitrary number. Moreover, the sums

$$
S_{m s N}(t, x)=\sum_{k=1}^{N} \xi_{k} e^{-\lambda_{k} t} \lambda_{k}^{m} X_{k}^{(s)}, \quad N=1,2, \ldots,
$$

are also strictly Orlicz processes.

Lemma 4.1. Let $\varepsilon$ be an arbitrary positive number. The series $S_{m s}(t, x)$ converge with probability one uniformly in the domain $D_{\varepsilon}=[0 \leq x \leq \pi] \times[\varepsilon, \infty)$.

Proof. According to [8] we have $\sup _{k=1, \infty} \sup _{0 \leq x \leq \pi}\left|X_{k}(x)\right| \leq C_{x}$, where $C_{x}$ is a constant and where

$$
\begin{gathered}
X_{n}(x)=\lambda_{n} \int_{0}^{\pi} G(x, s) X_{n}(s) \rho(s) d s, \\
G(x, s)= \begin{cases}u(x) v(s), & x \leq s, \\
u(s) v(x), & x>s .\end{cases}
\end{gathered}
$$

Here $u(x)$ and $v(x), x \in[0, \pi]$, are some twice continuously differentiable functions for $x \in[0, \pi]$. Hence

$$
\begin{gathered}
X_{n}^{\prime}(x)=\lambda_{n} \int_{0}^{\pi} G^{*}(x, s) X_{n}(s) \rho(s) d s, \\
X_{n}^{\prime \prime}(x)=\lambda_{n}\left(\int_{0}^{\pi} G^{* *}(x, s) X_{n}(s) \rho(s) d s+\left(v^{\prime}(x) u(x)-v(x) u^{\prime}(x)\right) X_{n}(x)\right),
\end{gathered}
$$

where

$$
\begin{aligned}
G^{*}(x, s) & = \begin{cases}u^{\prime}(x) v(s) & \text { if } x \leq s, \\
u(s) v^{\prime}(x) & \text { if } x>s,\end{cases} \\
G^{* *}(x, s) & = \begin{cases}u^{\prime \prime}(x) v(s) & \text { if } x \leq s, \\
u(s) v^{\prime \prime}(x) & \text { if } x>s\end{cases}
\end{aligned}
$$

Thus

$$
\left|X_{n}^{\prime}(x)\right| \leq \lambda_{n} \int_{0}^{\pi}\left|G^{*}(x, s)\right| \cdot\left|X_{n}(s)\right| \rho(s) d s \leq \lambda_{n} C_{x} \int_{0}^{\pi}\left|G^{*}(x, s)\right| d s \leq C^{*} \lambda_{n},
$$

where $C^{*}$ is a constant and where $\left|X_{n}^{\prime \prime}(x)\right| \leq \lambda_{n} C^{* *}$ for some constant $C^{* *}>0$. Hence

$$
\begin{aligned}
\sup _{\substack{t>\varepsilon \\
0 \leq x \leq \pi}} S_{m s}(t, x) & \leq \sum_{k=1}^{\infty}\left|\xi_{k}\right| \sup _{t>\varepsilon} e^{-\lambda_{k} t} \lambda_{k}^{m}\left|X_{k}^{(s)}(x)\right| \\
& \leq \hat{C} \sum_{k=1}^{\infty}\left|\xi_{k}\right| e^{-\lambda_{k} \varepsilon} \lambda_{k}^{m+1}
\end{aligned}
$$

where we set $\hat{C}=\max \left(C^{*}, C^{* *}, 1\right)$. The latter series converges with probability one if the series $\sum_{k=1}^{\infty} \mathrm{E}\left|\xi_{k}\right| e^{-\lambda_{k} \varepsilon} \lambda_{k}^{m+1}$ converges. Since

$$
\begin{aligned}
\mathrm{E}\left|\xi_{k}\right| & \leq\left(\mathrm{E} C_{k}^{2}\right)^{1 / 2}=\left(\mathrm{E}\left(\int_{0}^{\pi} X_{k}(x) \xi(x) \rho(x) d x\right)^{2}\right)^{1 / 2} \\
& =\left(\int_{0}^{\pi} \int_{0}^{\pi} X_{k}(x) X_{k}(y) \mathrm{E} \xi(x) \xi(y) \rho(x) \rho(y) d x d y\right)^{1 / 2} \\
& =\left(\int_{0}^{\pi} \int_{0}^{\pi} X_{k}(x) X_{k}(y) B(x, y) \rho(x) \rho(y) d x d y\right)^{\frac{1}{2}} \leq b
\end{aligned}
$$


where $b$ is a constant, we obtain

$$
\sum_{k=1}^{\infty} \mathrm{E}\left|\xi_{k}\right| e^{-\lambda_{k} \varepsilon} \lambda_{k}^{m+1} \leq b \sum_{k=1}^{\infty} e^{-\lambda_{k} \varepsilon} \lambda_{k}^{m+1} .
$$

According to 8 ,

$$
\sqrt{\lambda_{n}}=d n+O\left(\frac{1}{n}\right)
$$

where $d$ is a positive constant. Thus

$$
\sqrt{\lambda_{n}} \leq d_{1} n, \quad \sqrt{\lambda_{n}} \geq d_{2} n
$$

for sufficiently large $n$, where $0<d_{2}<d_{1}$ are some constants. Thus series (23) converges, since so does the series

$$
\sum_{k=1}^{\infty} e^{-d_{2}^{2} \varepsilon k^{2}} k^{2(m+1)} .
$$

Lemma 4.2. Let $T=[0, \infty)$ and let a function $X_{\lambda}(u), \lambda>0, u \in T$, be such that

1) $\sup _{u \in T}\left|X_{\lambda}(u)\right| \leq B$

2) $\left|X_{\lambda}(u)-X_{\lambda}(v)\right| \leq C \lambda|u-v|$ for all $u, v \in T$.

Let $\varphi(\lambda), \lambda>0$, be a continuous increasing function such that $\varphi(\lambda)>0$ for all $\lambda>0$ and the function $\lambda / \varphi(\lambda)$ is increasing for $\lambda>v_{0}$ for some constant $v_{0} \geq 0$. Then

$$
\left|X_{\lambda}(u)-X_{\lambda}(v)\right| \leq \max (C ; 2 B) \frac{\varphi\left(\lambda+v_{0}\right)}{\varphi\left(|u-v|^{-1}+v_{0}\right)}
$$

for all $\lambda \geq 0$ and $v>0$.

Proof. Let $\lambda \geq|u-v|^{-1}$. Then

$$
\frac{\left|X_{\lambda}(u)-X_{\lambda}(v)\right|}{2 B} \leq 1 \leq \frac{\varphi\left(\lambda+v_{0}\right)}{\varphi\left(|u-v|^{-1}+v_{0}\right)} ;
$$

that is, inequality (24) holds.

Now let $\lambda \leq|u-v|^{-1}$. Since $\lambda / \varphi(\lambda)$ is an increasing function for $\lambda>v_{0}$, we get

$$
\begin{aligned}
\left|X_{\lambda}(u)-X_{\lambda}(v)\right| & \leq C \lambda|u-v| \leq C \frac{\lambda+v_{0}}{|u-v|^{-1}+v_{0}} \\
& \leq C \frac{\varphi\left(\lambda+v_{0}\right)}{\varphi\left(|u-v|^{-1}+v_{0}\right)} ;
\end{aligned}
$$

that is, inequality (24) holds in this case, too.

Lemma 4.3. Let $\xi(x)$ be a continuous with probability one process on $[0, T]$. Assume that

$$
\mathrm{E}|V(t, x)-\xi(x)|^{2} \rightarrow 0 \quad \text { as } t \rightarrow 0
$$

for all $0 \leq x \leq \pi$ and that there exists a continuous increasing function $\sigma=(\sigma(h), 0<$ $h \leq \pi)$ such that $\sigma(h) \rightarrow 0$ as $h \rightarrow 0$ and

$$
\sup _{0<t<t_{0}} \sup _{\substack{|x-y| \leq h \\ x, y \in[0, \pi]}}\left(\mathrm{E}|V(t, x)-V(t, y)|^{2}\right)^{1 / 2} \leq \sigma(h),
$$

where $0<t_{0}<T$. If

$$
\int_{0}^{\varepsilon} U^{(-1)}\left(\frac{\pi}{2 \sigma^{(-1)}(h)}+1\right) d h<\infty
$$

for all $\varepsilon>0$, then the series $V(t, x)$ converges in probability to $\xi(x)$ as $t \rightarrow 0$ uniformly in the interval $[0, \pi]$. 
Proof. Since $V(t, x)$ is a strictly Orlicz process, we obtain

$$
\|V(t, x)-V(t, y)\| \leq C_{\Delta}\left(\mathrm{E}(V(t, x)-V(t, y))^{2}\right)^{1 / 2} .
$$

Thus Lemma 4.3 follows from Theorem 3.3, since $V(t, x) \rightarrow \xi(x)$ in probability.

Remark 4.1. Relation (26) holds if $\sigma(h)=C h$ in condition (25).

Proof of Remark 4.1. If $U(x) \succ x^{2}$, then

$$
U(x) \geq C x^{2}
$$

for sufficiently large $x>0$, namely for $x$ such that

$$
x \geq U^{(-1)}\left(C x^{2}\right) .
$$

This means that

$$
U^{(-1)}(t) \leq \sqrt{\frac{t}{C}}
$$

for sufficiently large $t$. Thus

$$
\begin{aligned}
& \int_{0}^{\varepsilon} U^{(-1)}\left(\frac{\pi}{2 \sigma^{(-1)}(h)}+1\right) d h=\int_{0}^{\varepsilon} U^{(-1)}\left(\frac{C \pi C}{2 h}+1\right) d h \\
& \quad \leq \int_{0}^{\varepsilon} \sqrt{\frac{\pi}{2 h}+\frac{1}{C}} d h \leq \int_{0}^{\varepsilon} \sqrt{\frac{\pi}{2 h}} d h+\frac{1}{C} \int_{0}^{\varepsilon} d h=\sqrt{2 \pi \varepsilon}+\frac{\varepsilon}{C}<\infty
\end{aligned}
$$

for sufficiently small $\varepsilon$, since

$$
\sigma^{(-1)}(h)=\frac{h}{C}
$$

Lemma 4.4. Let $\xi(x), 0 \leq x \leq \pi$, be a strictly Orlicz stochastic process. Assume that the assumptions of Theorem 2.1 hold. Let $\varphi(x)$ be a function such that the assumptions of Lemma 4.2 hold and let

$$
\int_{0}^{\varepsilon} U^{(-1)}\left(\frac{\pi}{2}\left(\varphi^{(-1)}\left(\frac{c}{h}\right)-v_{0}\right)+1\right) d h<\infty,
$$

where $c>0$ is an arbitrary constant. If

$$
\sum_{k=1}^{\infty} \sum_{l=1}^{\infty}\left|\mathrm{E} \xi_{k} \xi_{l}\right| \varphi\left(\lambda_{k}+v_{0}\right) \varphi\left(\lambda_{l}+v_{0}\right)<\infty
$$

then

$$
V(t, x) \rightarrow \xi(x) \quad \text { as } t \rightarrow 0
$$

in probability and uniformly in the interval $[0, \pi]$.

Proof. We derive Lemma 4.4 from Lemma 4.3 First we check the assumptions of Lemma 4.3 .

Note that the assumptions of Lemma 4.4 imply that

$$
\mathrm{E}|V(t, x)-\xi(x)|^{2} \rightarrow 0 \quad \text { as } t \rightarrow 0 .
$$


Indeed,

$$
\begin{aligned}
\mathrm{E}|V(t, x)-\xi(x)|^{2} & =\mathrm{E}\left(\sum_{k=1}^{\infty} \xi_{k} e^{-\lambda_{k} t} X_{k}(x)-\xi_{k} X_{k}(x)\right)^{2} \\
& =\mathrm{E}\left(\sum_{k=1}^{\infty} \xi_{k} X_{k}(x)\left(e^{-\lambda_{k} t}-1\right)\right)^{2} \\
& =\sum_{k=1}^{\infty} \sum_{l=1}^{\infty} \mathrm{E} \xi_{k} \xi_{l} X_{k}(x) X_{l}(x)\left(e^{-\lambda_{k} t}-1\right)\left(e^{-\lambda_{l} t}-1\right),
\end{aligned}
$$

since $\xi(x)=\sum_{k=1}^{\infty} \xi_{k} X_{k}(x)$. The function $X_{\lambda}(t)=e^{-\lambda t}$ satisfies the assumptions of Lemma 4.2 with $B=1$ and $C=1$. Since $\left|X_{k}(x)\right| \leq C_{x}$, we get

$$
\begin{aligned}
& \mathrm{E}|V(t, x)-\xi(x)|^{2} \\
& \quad \leq \sum_{k=1}^{\infty} \sum_{l=1}^{\infty}\left|\mathrm{E} \xi_{k} \xi_{l} X_{k}(x) X_{l}(x)\right| \varphi\left(\lambda_{k}+v_{0}\right) \varphi\left(\lambda_{l}+v_{0}\right) \varphi^{-2}\left(\frac{1}{t}+v_{0}\right) \\
& \quad \leq C_{X}^{2} \sum_{k=1} \sum_{l=1}\left|\mathrm{E} \xi_{k} \xi_{l}\right| \varphi\left(\lambda_{k}+v_{0}\right) \varphi\left(\lambda_{l}+v_{0}\right) \varphi^{-2}\left(\frac{1}{t}+v_{0}\right) \rightarrow 0 \quad \text { as } t \rightarrow 0 .
\end{aligned}
$$

Now we find a function $\sigma(h)$ such that

$$
\sup _{0<t<t_{0}} \sup _{\substack{\left|x-x_{1}\right| \leq h \\ x_{1}, x_{2} \in[0, \pi]}}\left(\mathrm{E}\left|V(t, x)-V\left(t, x_{1}\right)\right|^{2}\right)^{1 / 2} \leq \sigma(h) .
$$

It is easy to see that

$$
\begin{aligned}
& \mathrm{E}\left(V(t, x)-V\left(t, x_{1}\right)\right)^{2} \\
& \quad=\mathrm{E} \sum_{k=1}^{\infty}\left(\xi_{k} X_{k}(x) e^{-\lambda_{k} t}-\xi_{k} X_{k}\left(x_{1}\right) e^{-\lambda_{k} t}\right)^{2} \\
& \quad=\mathrm{E}\left(\sum_{k=1}^{\infty} e^{-\lambda_{k} t}\left(\xi_{k}\left(X_{k}(x)-X_{k}\left(x_{1}\right)\right)\right)\right)^{2} \\
& \quad=\sum_{k=1}^{\infty} \sum_{l=1}^{\infty} e^{-\lambda_{k} t} e^{-\lambda_{l} t}\left(\mathrm{E} \xi_{k} \xi_{l}\right)\left(X_{k}(x)-X_{k}\left(x_{1}\right)\right)\left(X_{l}(x)-X_{l}\left(x_{1}\right)\right) \\
& \leq \sum_{k=1}^{\infty} \sum_{l=1}^{\infty} e^{-\lambda_{k} t} e^{-\lambda_{l} t}\left|\mathrm{E} \xi_{k} \xi_{l}\right| \cdot\left|X_{k}(x)-X_{k}\left(x_{1}\right)\right| \cdot\left|X_{l}(x)-X_{l}\left(x_{1}\right)\right| \\
& \leq \sum_{k=1}^{\infty} \sum_{l=1}^{\infty}\left|\mathrm{E} \xi_{k} \xi_{l}\right| \cdot\left|X_{k}(x)-X_{k}\left(x_{1}\right)\right| \cdot\left|X_{l}(x)-X_{l}\left(x_{1}\right)\right|, \\
& \quad X_{k}(x)=\lambda_{k} \int_{0}^{\pi} G(x, y) X_{k}(y) \rho(y) d y, \\
& G(x, s)= \begin{cases}u(x) v(y), & \text { if } x \leq y, \\
u(y) v(x), & \text { if } x>y,\end{cases}
\end{aligned}
$$


and

$$
\begin{aligned}
\left|X_{k}(x)-X_{k}\left(x_{1}\right)\right| & =\left|\lambda_{k} \int_{0}^{\pi}\left(G(x, y)-G\left(x_{1}, y\right)\right) X_{k}(y) \rho(y) d y\right| \\
& \leq \lambda_{k} \int_{0}^{\pi}\left|G(x, y)-G\left(x_{1}, y\right)\right| \cdot\left|X_{k}(y)\right| \rho(y) d y \\
& \leq \lambda_{k} \widetilde{C}_{X} \int_{0}^{\pi}\left|G(x, y)-G\left(x_{1}, y\right)\right| \rho(y) d y
\end{aligned}
$$

where

$$
\widetilde{C}_{X}=C_{X} \sup \rho(x)
$$

Let

$$
\begin{array}{ll}
C_{v}^{\prime}=\sup _{0 \leq y \leq \pi}\left|v^{\prime}(y)\right|, & C_{u}^{\prime}=\sup _{0 \leq x \leq \pi}\left|u^{\prime}(x)\right|, \\
C_{v}=\sup _{0 \leq y \leq \pi}|v(y)|, & C_{u}=\sup _{0 \leq x \leq \pi}|u(x)| .
\end{array}
$$

Consider the integral

$$
\begin{aligned}
I= & \int_{0}^{\pi}\left|G(x, y)-G\left(x_{1}, y\right)\right| d y \\
= & \int_{0}^{x_{1}}\left|G(x, y)-G\left(x_{1}, y\right)\right| d y+\int_{x_{1}}^{x}\left|G(x, y)-G\left(x_{1}, y\right)\right| d y \\
& +\int_{x}^{\pi}\left|G(x, y)-G\left(x_{1}, y\right)\right| d y \\
= & I_{1}+I_{2}+I_{3},
\end{aligned}
$$

where

$$
\begin{gathered}
I_{1}=\int_{0}^{x_{1}}\left|u(y)\left(v(x)-v\left(x_{1}\right)\right)\right| d y \leq \int_{0}^{x_{1}} C_{u} C_{v}^{\prime}\left|x-x_{1}\right| d y \leq \pi C_{v} C_{u}^{\prime}\left|x-x_{1}\right|, \\
I_{2} \leq \int_{x_{1}}^{x}\left(\sup _{0 \leq y \leq \pi}|u(y)| \sup _{0 \leq x \leq \pi}|v(x)|+\sup _{0 \leq x \leq \pi}|u(x)| \sup _{0 \leq y \leq \pi}|v(y)|\right) d y \leq 2 C_{u} C_{v}\left|x-x_{1}\right|, \\
I_{3}=\int_{x}^{\pi}\left|v(y)\left(u(x)-u\left(x_{1}\right)\right)\right| d y \leq \pi C_{v} C_{u}^{\prime}\left|x-x_{1}\right|,
\end{gathered}
$$

that is,

$$
I \leq\left|x-x_{1}\right|\left(C_{v} C_{u}^{\prime}+2 C_{u} C_{v}+C_{u} C_{v}\right) \leq\left|x-x_{1}\right| K,
$$

where $K$ is a constant.

We have proved that

$$
\left|X_{k}(x)-X_{k}\left(x_{1}\right)\right| \leq D\left|x-x_{1}\right| \lambda_{k},
$$

where $D=\left(C_{v} C_{u}^{\prime}+2 C_{u} C_{v}+C_{u} C_{v}^{\prime}\right) C_{X}$.

The estimate $\left|X_{k}(x)\right| \leq C_{X}$ and Lemma 4.2 imply that

$$
\left|X_{k}(x)-X_{k}\left(x_{1}\right)\right| \leq \max \left(2 C_{X}, D\right) \cdot \frac{\varphi\left(\lambda_{k}+v_{0}\right)}{\varphi\left(\left|x-x_{1}\right|^{-1}+v_{0}\right)},
$$

whence

$$
\begin{aligned}
\mathrm{E}(V(t, x)-V(t, y))^{2} & =\sum_{k=1}^{\infty} \sum_{l=1}^{\infty}\left(\mathrm{E} \xi_{k} \xi_{l}\right)\left(X_{k}(x)-X_{k}(y)\right)\left(X_{l}(x)-X_{l}(y)\right) \\
& \leq \sum_{k=1}^{\infty} \sum_{l=1}^{\infty}\left|\mathrm{E} \xi_{k} \xi_{l}\right| 4 D^{2} \varphi\left(\lambda_{k}+v_{0}\right) \varphi\left(\lambda_{l}+v_{0}\right) \varphi^{-2}\left(\frac{1}{|x-y|}+v_{0}\right)
\end{aligned}
$$


Thus

$$
\sigma(h)=C\left(\varphi\left(\frac{1}{h}+v_{0}\right)\right)^{-1}
$$

and

$$
\int_{0}^{\varepsilon} U^{(-1)}\left(\frac{\pi}{2 \sigma^{(-1)}(h)}+1\right) d h<\infty
$$

in view of

$$
\sigma^{(-1)}(h)=\frac{1}{\varphi^{(-1)}(C / h)-v_{0}} .
$$

According to Theorem 3.3, condition (8) implies that the process $\xi(x)$ is continuous with probability one. Thus all the assumptions of Lemma 4.3 are satisfied.

Lemma 4.5. Let $X_{k}(x), 0 \leq x \leq \pi$, be defined as above. Assume that

$$
\left|X_{k}(x)-X_{k}\left(x_{1}\right)\right| \leq L \lambda_{k}\left|x-x_{1}\right|
$$

for $0 \leq x \leq \pi$. (We have shown in the proof of Lemma 4.4 that $L=D$, where $D$ is defined by (32), although the values of the constant $D$ can be estimated more accurately in several particular cases.) Assume that the series

$$
\sum_{k=1}^{\infty} \sum_{l=1}^{\infty}\left|\mathrm{E} \xi_{k} \xi_{l}\right| \cdot \varphi\left(\lambda_{k}+v_{0}\right) \varphi\left(\lambda_{l}+v_{0}\right)=W
$$

converges, where the function $\varphi$ is defined in Lemma 4.2. Then

$$
\sup _{\max \left(\left|t-t_{1}\right|,\left|x-x_{1}\right|\right) \leq h}\left(\mathrm{E}\left(z(t, x)-z\left(t_{1}, x_{1}\right)\right)^{2}\right)^{1 / 2} \leq \frac{\sqrt{2 \max \left(2 C_{X}, L\right)}}{\varphi\left(1 / h+v_{0}\right)} \sqrt{W}
$$

for $t_{1} \geq t \geq 0$ and $0 \leq x, x_{1} \leq \pi$, where $C_{X}$ is a constant such that $X_{k}(x)<C_{X}$ and where $z(t, x)=V(t, x)-\xi(x)$.

Proof. It is easy to see that

$$
\begin{aligned}
& z(t, x)-z\left(t_{1}, x_{1}\right)=\sum_{k=1}^{\infty} \xi_{k}\left(X_{k}(x)\left(e^{-\lambda_{k} t}-1\right)-X_{k}\left(x_{1}\right)\left(e^{-\lambda_{k} t_{1}}-1\right)\right), \\
& \mathrm{E}\left(z(t, x)-z\left(t_{1}, x_{1}\right)\right)^{2}=\sum_{k=1}^{\infty} \sum_{l=1}^{\infty} \mathrm{E} \xi_{k} \xi_{l} \cdot a_{k} a_{l} \leq \sum_{k=1}^{\infty} \sum_{l=1}^{\infty}\left|\mathrm{E} \xi_{k} \xi_{l}\right| \cdot\left|a_{k}\right| \cdot\left|a_{l}\right|,
\end{aligned}
$$

where

$$
a_{k}=\left(X_{k}(x)\left(e^{-\lambda_{k} t}-1\right)-X_{k}\left(x_{1}\right)\left(e^{-\lambda_{k} t_{1}}-1\right)\right)
$$

Note that

$$
\begin{aligned}
\left|a_{k}\right| & \leq\left|X_{k}(x)-X_{k}\left(x_{1}\right)\right| \cdot\left|e^{-\lambda_{k} t}-1\right|+\left|X_{k}\left(x_{1}\right)\right| \cdot\left|e^{-\lambda_{k} t}-e^{-\lambda_{k} t_{1}}\right| \\
& \leq\left|X_{k}(x)-X_{k}\left(x_{1}\right)\right|+C_{x}\left|e^{-\lambda_{k} t}-e^{-\lambda_{k} t_{1}}\right| .
\end{aligned}
$$

Lemma 4.2 implies that

$$
\left|X_{k}(x)-X_{k}\left(x_{1}\right)\right| \leq \max \left(2 C_{x}, L\right) \cdot \frac{\varphi\left(\lambda_{k}+v_{0}\right)}{\varphi\left(\left|x-x_{1}\right|^{-1}+v_{0}\right)} .
$$

Since the function $e^{-\lambda t}$ satisfies the assumptions of Lemma 4.2 with $C=1$ and $B=1$,

$$
\left|a_{k}\right| \leq 2 \max \left(2 C_{x}, L\right) \frac{\varphi\left(\lambda_{k}+v_{0}\right)}{\varphi\left(1 / h+v_{0}\right)},
$$

where $h=\max \left(\left|t-t_{1}\right|,\left|x-x_{1}\right|\right)$ and Lemma 4.5 follows from (36). 
Proof of Theorem 2.1. The almost sure uniform convergence of the series $S_{m s}(t, x)$ with respect to $0 \leq x \leq \pi$ and $0<t<\varepsilon$ is proved in Lemma 4.1 It follows from Lemma 4.4 that if condition (10) holds, then

$$
V(t, x) \rightarrow \xi(x) \text { as } t \rightarrow 0
$$

in probability and uniformly in the interval $[0, \pi]$. Now we prove that conditions (11) and (12) imply that

$$
V(t, x) \rightarrow \xi(x) \quad \text { as } t \rightarrow 0
$$

with probability one and uniformly in the interval $0 \leq x \leq \pi$. Consider the stochastic process $z(t, x)=V(t, x)-\xi(x)$ in the domain $0 \leq t, t_{1} \leq \hat{t} \leq \pi$. Lemma 4.5 implies that

$$
\begin{aligned}
\sup _{\max \left(\left|t-t_{1}\right|,\left|x-x_{1}\right|\right) \leq h}\left\|z(t, x)-z\left(t_{1}, x_{1}\right)\right\|_{L_{U}} \\
\quad \leq C_{\Delta} \sup _{\max \left(\left|t-t_{1}\right|,\left|x-x_{1}\right|\right) \leq h}\left(\mathrm{E}\left(z(t, x)-z\left(t_{1}, x_{1}\right)\right)^{2}\right)^{1 / 2} \leq R \varphi\left(\frac{1}{h}+v_{0}\right),
\end{aligned}
$$

where $R=C_{\Delta} \sqrt{W} \sqrt{2 \max \left(2 C_{X}, L\right)}$. Thus the assumptions of Corollary 3.1 hold for the process $z(t, x)$, since $\sigma(h)=R \varphi\left(1 / h+v_{0}\right)$, whence

$$
\begin{aligned}
\int_{0}^{\varepsilon} & U^{(-1)}\left(\left(\frac{b}{2 \sigma^{(-1)}}+1\right)\left(\frac{d-c}{2 \sigma^{(-1)}}+1\right)\right) d u \\
& =\int_{0}^{\varepsilon} U^{(-1)}\left(\left(\frac{\pi}{2}\left(\varphi^{(-1)}\left(\frac{R}{u}\right)-v_{0}\right)+1\right)\left(\frac{\hat{t}}{2}\left(\varphi^{(-1)}\left(\frac{R}{u}\right)-v_{0}\right)+1\right)\right) d u \\
& \leq \int_{0}^{\varepsilon} U^{(-1)}\left(\left(\frac{\pi}{2}\left(\varphi^{(-1)}\left(\frac{R}{u}\right)-v_{0}\right)+1\right)^{2}\right) d u<\infty .
\end{aligned}
$$

Therefore

$$
\mathrm{P}\left\{\sup _{0 \leq x \leq \pi, 0 \leq t \leq \hat{t}}|z(t, x)|>y\right\} \leq\left(U\left(\frac{y}{\tilde{B}(\theta)}\right)\right)^{-1}
$$

for a sufficiently small $\theta$, where

$$
\tilde{B}(\theta)=C_{\Delta}\left(\mathrm{E}(z(0,0))^{2}\right)^{1 / 2}+\frac{1}{\theta(1-\theta)} \int_{0}^{s \theta} U^{(-1)}\left(\left(\frac{\pi}{2}\left(\varphi^{(-1)}\left(\frac{R}{u}\right)-v_{0}\right)+1\right)^{2}\right) d u
$$

$s=2 \sup _{t \in V_{\hat{t}}}\|z(t, x)\|_{L_{U}}$, and $V_{\hat{t}}=\{0 \leq x \leq \pi, 0 \leq t \leq \hat{t}\}$. Since

$$
\|z(t, x)\|_{L_{U}} \leq C_{\Delta} \sqrt{\mathrm{E}(z(t, x))^{2}},
$$

relation (30) implies that

where

$$
\|z(t, x)\|_{L_{U}} \leq g \frac{1}{\varphi\left(1 / t+v_{0}\right)},
$$

$$
g=C_{\Delta} C_{X}\left(\sum_{k=1}^{\infty} \sum_{l=1}^{\infty}\left|\mathrm{E} \xi_{k} \xi_{l}\right|\right) \cdot \varphi\left(\lambda_{k}+v_{0}\right) \varphi\left(\lambda_{l}+v_{0}\right)^{1 / 2}
$$

and $\mathrm{E}(z(0,0))^{2}=0$.

Thus $\tilde{B}(\theta) \rightarrow 0$ as $\hat{t} \rightarrow 0$, whence

$$
\mathrm{P}\left\{\sup _{0 \leq x \leq \pi, 0 \leq t \leq \hat{t}}|Z(t, x)|>\varepsilon\right\} \underset{\hat{t} \rightarrow 0}{\longrightarrow} 0,
$$

that is,

$$
\sup _{0 \leq x \leq \pi, 0 \leq t \leq \hat{t}}|Z(t, x)| \underset{\hat{t} \rightarrow 0}{\longrightarrow} 0
$$


in probability. Since the function $\sup _{0 \leq x \leq \pi, 0 \leq t \leq \hat{t}}|Z(t, x)|$ decreases with $\hat{t}$, we have proved that

$$
\sup _{0 \leq x \leq \pi, 0 \leq t \leq \hat{t}}|Z(t, x)| \underset{\hat{t} \rightarrow 0}{\longrightarrow} 0
$$

with probability one, that is, $V(t, x) \rightarrow \xi(x)$ with probability one and uniformly in $0 \leq x \leq \pi$.

Proof of Theorem 2.2. Theorem 2.2 follows from Corollary 3.1 and from the proof of Theorem 2.1 by putting $\hat{t}_{0}=(0,0)$ in Corollary 3.1 .

\section{Concluding Remarks}

Conditions justifying an application of the Fourier method for parabolic equations with initial conditions that are stochastic processes belonging to the Orlicz spaces of random variables are obtained in the paper. Some bounds for the distribution of the supremum of solutions of such equations are found.

\section{BIBLIOGRAPHY}

1. V. V. Buldygin and Yu. V. Kozachenko, On an applicability of the Fourier method for solving problems with random boundary conditions, Random Processes in the Problems of Mathematical Physics, Institute of Mathematics, Academy of Science of Ukr. SSR, Kiev, 1979, pp. 4-35. (Russian) MR587658 (83i:35080)

2. E. Beĭsenbaev and Yu. V. Kozachenko, Uniform convergence of random series in probability and the solution of boundary-value problems with random initial conditions, Teor. Imovir. Mat. Stat. 21 (1979), 9-23; English transl. in Theory Probab. Math. Statist. 21 (1980), 9-24. MR550238 (81g:60036)

3. V. V. Buldygin and Yu. V. Kozachenko, Metric Characterization of Random Variables and Random Processes, TViMS, Kiev, 1998; English transl., Translations of Math. Mono., vol. 188, American Mathematical Society, Providence, RI, 2000. MR 1743716 (2001g:60089)

4. B. V. Dovgay, Yu. V. Kozachenko, and G. I. Slyvka-Tylyshchak, Boundary-Value Problems of Mathematical Physics with Random Factors, Kyiv University, Kyiv, 2008. (Ukrainian)

5. E. Barrasa de la Krus and Yu. V. Kozachenko, Boundary-value problems for equations of mathematical physics with strictly Orlicz random initial conditions, Random Oper. Stoch. Eq. 3 (1995), no. 3, 201-220. MR.1354813 (96h:60009)

6. Yu. V. Kozachenko and G. I. Slivka, Justification of the Fourier method for hyperbolic equations with random initial conditions, Teor. Imovir. Mat. Stat. 69 (2003), 63-78; English transl. in Theory Probab. Math. Statist. 69 (2004), 67-83. MR.2110906 (2005k:60127)

7. Yu. V. Kozachenko and M. M. Perestyuk, On the uniform convergence of wavelet expansions of random processes belonging to the Orlicz spaces of random variables. I, Ukr. Matem. Zh. 59 (2007), no. 12, 1647-1659; English transl. in Ukrain. Math. J. 59 (2007), no. 12, 1850-1869. MR22411593 (2009b:60058)

8. I. G. Polozhyı̆, Equations of Mathematical Physics, Vysshaya Shkola, Moscow, 1964. (Russian)

Department of Probability Theory and Mathematical Statistics, Faculty for Mechanics and Mathematics, National Taras Shevchenko University, Academician Glushkov Avenue, 2, KIEV 03127, UKRAINe

E-mail address: yvk@univ.kiev.ua

Department of Probability Theory and Mathematical Statistics, Faculty for Mechanics and Mathematics, National Taras Shevchenko University, Academician Glushkov Avenue, 2, Kiev 03127, UKRAINE

E-mail address: yvk@univ.kiev.ua

Received 2/FEB/2009

Translated by OLEG KLESOV 